\title{
Placement and Sizing of DG Using PSO\&HBMO Algorithms in Radial Distribution Networks
}

\author{
M.Afzalan \\ Department of Electrical and Computer Engineering, Saveh Branch, Islamic Azad University, Saveh, Iran \\ m_power1366@yahoo.com \\ M. A.Taghikhani \\ Department of Engineering, Imam Khomeini International University, Qazvin, Iran \\ taghikhani@ikiu.ac.ir
}

\begin{abstract}
Optimal placement and sizing of DG in distribution network is an optimization problem with continuous and discrete variables. Many researchers have used evolutionary methods for finding the optimal DG place ment and sizing. This paper proposes a hybrid algorithm PSO\&HBMO for optimal placement and sizing of distributed generation (DG) in radial distribution system to minimize the total power loss and improve the voltage profile. The proposed method is tested on a standard 13 bus radial distribution system and simulation results carried out using MATLAB software. The simulation results indicate that PSO\&HBMO method can obtain better results than the simp le heuristic search method and PSO algorithm. The method has a potential to be a tool for identifying the best location and rating of a DG to be installed for improving voltage profile and line losses reduction in an electrical power system. Moreover, current reduction is obtained in distribution system.
\end{abstract}

Index Terms - Distributed Generation; Distribution Networks; Particle Swarm Optimization (PSO); Sensitivity Analysis; Honey Bee Mating Optimization (HBMO); Voltage Profile

\section{Introduction}

One of the most important motivation for the studies on integration of distributed resources to the grid is the exploitation of the renewable resources such as; hydro, wind, solar, geothermal, biomass and ocean energy, which are naturally scattered around the country and also smaller in size. Accordingly, these resources can only be tapped through integration to the distribution system by means of distributed generation. Distributed generation (DG), which generally consists of various types of renewable resources, can be defined as electric powergeneration with in distribution networks or on the customer side of the system [1].DG affects the flow of power and voltage conditions on the system equip ment. These impacts may manifest themselves either positively or negatively depending on the distribution system operating conditions and the DG characteristics.
Positive impacts are generally called 'system support benefits', and include voltage support and improved power quality; loss reduction; transmission and distribution capacity release; improved utility system reliability. On account of achieving above benefits, the DG must be reliable of the proper size and at the proper locations [2], [3]. Distributed Generation is a s mall generator spotted throughout a power system network, providing the electricity locally to load customers. DG can be an alternative for industrial, commercial and residential applications. DG makes use of the latest modern technology which is efficient, reliable, and simple enough so that it can compete with traditional large generators in some areas [4], [5]. In fact, one type of DG is capable of supplying real power and reactive power.

A stochastic dynamic multi-objective model for integration of DG in distribution networks is proposed in [6] with a binary PSO algorith $\mathrm{m}$. A distribution system expansion planning strategy encompassing renewable DG systems with schedulable and intermittent power generation patterns is presented in [7] that a solution algorith $m$ integrating TRIBE PSO and ordinal optimization (OO) is developed to obtain optimal and near-optimal solutions for system planners. A DG interconnection planning study framework is brought in [8] that includes a coordinated feeder reconfiguration and voltage control to calculate the maximu m allowable DG capacity at a given node in the distribution network. In [9] a distributed micro-grid planning model has been presented to optimize the locating and the unit capacities with in DG micro-grid, in which wind power and photovoltaic power are taken into consideration simultaneously with both Elitis m Genetic Algorithm (EGA) and PSO.

A multi-objective index-based approach for optimally determining the size and location of multi-distributed generation (multi-DG) units in distribution systems with different load models based on PSO is introduced in $[10,11]$ and a combined genetic algorithm $(\mathrm{GA}) /(\mathrm{PSO})$ is presented in [12] for optimal location and sizing of DG on distribution systems. A population-based heuristic approach for optimal loca 
tion and capacity of DGs in distribution networks, with the objectives of minimization of fuel cost, power loss reduction, and voltage profile imp rovement is proposed in [13] that the approach employs an improved group search optimizer (iGSO) by incorporating PSO into group search optimizer (GSO) for optimal setting of DGs. A new hybrid method which employs discrete PSO and optimal power flow is introduced in [14] which could apply to connect distributed generation systems in a distribution network choosing among a large number of potential combinations.

Therefore, in this paper, PSO\&HBMO method is proposed to determine the optimal location and sizes of multi-DGs to minimize the total power loss and improve the voltage profile of the distribution systems. The organization of this paper is as follows: The load flow, the problem formulation and sensitivity analysis are shown in Section II. The PSO algorithm is in Section III. The HBMO algorith $m$ is represented in Section IV. The proposed optimization method and PSO\&HBMO procedure for the optimal placement and sizing of distributed generation problem are given in Section V. Simulation results on the test systems are illustrated in Section VI.

\section{Load Flow}

On account of the some inherent features of distribution systems such as; radial structure, unbalanced distributed loads, large number of nodes, a wide range of $\mathrm{R} / \mathrm{X}$ ratios; the conventional techniques developed for transmission systems generally fail on the determination of optimum size and location of distributed generations. The backward/forward sweep load flow algorith $\mathrm{m}$ is used in this paper. The voltage for all buses firstly is considered with zero phases then knowing power of bus we can find current of each bus. We use the obtained new voltages to calculate the current of buses so as the permitted repeat time is reached. After finding the line currents we use the first voltage bus to calculate the voltage of the buses. The problem is to determine allocation and size of the DGs which min imizes the distribution power losses and improve the voltage profile for a fixed number of DGs and specific total capacity of the DGs. Therefore, the following assumptions are employed in this formulation:

Objective function is defined as cost over profit. Costs include charge of active and reactive power production and the advantage is obtained from reduction of losses and variance of voltage.

$$
\begin{aligned}
& \text { sub }=k p g *\left(p_{D G}+p_{\text {loss }}\right)+k q^{*}\left(q_{D G}+q_{\text {loss }}\right) / \\
& \left(\left(k p *^{*}\left(p_{\text {loss old }}-p_{\text {loss }}\right)+k q^{*}\left(q_{\text {loss old }}-q_{\text {loss }}\right)\right.\right. \\
& \left.+k v^{*}\left(v_{\text {cast old }}-v_{\text {cost }}\right)\right)
\end{aligned}
$$

$k p g$ is the real power production price. $k q$ is the reactive power price. $P_{\text {LOSS }}$ is the active power loss in presence of distributed generator. $P_{D G}$ is the active production power of distributed generator. $q_{D G}$ is the reactive production power of distributed generator. $q_{\text {LOSS }}$ is the reactive power loss in presence of distributed generator. $P_{\text {lossold }}$ is the active power loss before installation of distributed generator. $q_{\text {lossold }}$ is the reactive power before installation of distributed generator. $V_{\text {costold }}$ is the voltage variation from ideal condition before installation of distributed generator. The price of DG power is considered to be $313 \mathrm{ryal} / \mathrm{kw}$. Direct effect of reactive power on voltage profile $k v$ factor can be held equal to $k q$. $k p s$ factor is the price of real power which is referred to $354 \mathrm{ryal} / \mathrm{kw}$.

$$
\begin{aligned}
& \text { sub }=313 *\left(p_{D G}+p_{\text {loss }}\right)+82400 *\left(q_{D G}+q_{\text {loss }}\right) / \\
& \left(\left(354 *\left(336-p_{\text {loss }}\right)+82400 *\left(264-q_{\text {loss }}\right)\right.\right. \\
& \left.+82400 *\left(.45-v_{\text {cast }}\right)\right)
\end{aligned}
$$

The constraints are listed as follows:

a) Distribution line absolute power limits:

$$
\mid \text { PijLine } \mid \leq \text { Pij, maxLine }
$$

| PijLinel and Pij, maxLine are the absolute power and its corresponding maximum allowable value flowing over the distribution line between the nodes $i$ and $\mathrm{j}$, respectively.

\section{b) Bus voltage limit:}

Bus voltage amplitudes are limited as

$$
\mathrm{V} \min \leq \mathrm{Vi} \leq \mathrm{V} \max
$$

Where Vmin and Vmax are the minimum and maximum values of bus voltage amplitudes, respectively.

\section{c) Power limits of DG:}

PDGimin $\leq$ PDGi $\leq$ PDGimax,

QDGimin $\leq$ QDGi $\leq$ QDGimax

Where Pi and Qi are the injected active and reactive power of DG components at the ith bus.

\section{d) Subject to power balance constraints:}

$$
\sum_{i=1}^{N_{s c}} P_{D G i}=\sum_{i=1}^{N_{s c}} P_{D i}+P_{L}
$$

Where Nsc is total number of sections, PL is the real power loss in the system, PDGi is the real power generation at bus i, PDi is the power demand at bus $i$.

In this analysis, the amounts of active and reactive power are separately increased and in each increase the 
magnitude of objective function will be calcu lated. The bus which has most variation to minimize the objective function is determined as the suitable place to install the first DG. This method is used in [15]. The amount of DG will be calculated in the next step using proposed optimization algorith m. Fig. 1 shows that the best buses for DG installation are 2,11,4 respectively.

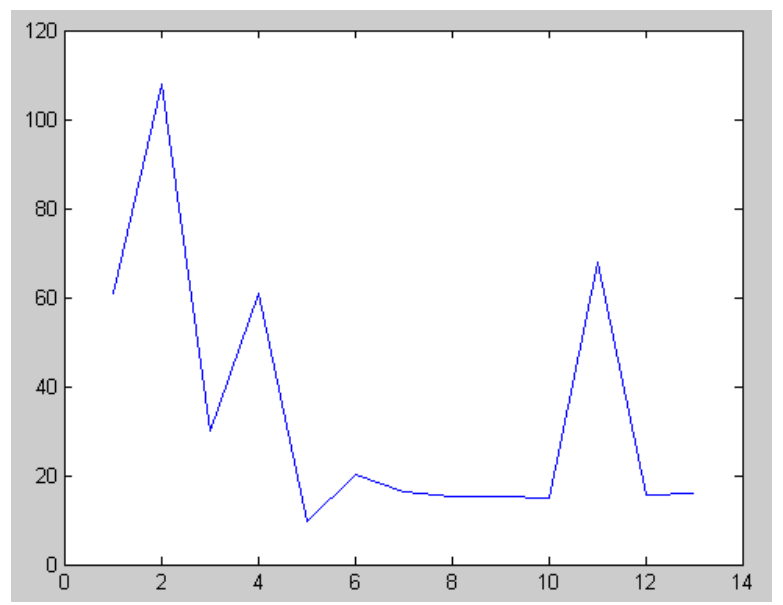

Fig.1 Sensitivity of objective function to the location of DG

\section{Particle Swarm Optimization}

PSO is a population-based, stochastic optimization algorith $m$ based on the idea of a swarm moving over a given landscape. The algorithm adaptively updates the velocities and members positions of the swarm by learning from the good experiences. In PSO, the velocity vid and position xid of the $d$ th dimension of the $i$ th particle are updated as follows:

$$
\begin{aligned}
& v_{i}^{d}=w \cdot v_{i}^{d}+c_{1} \cdot r_{1} \cdot\left(\text { pbest }_{i}^{d}-x_{i}^{d}\right) \\
& +c_{2} \cdot r_{2} \cdot\left(\text { gbest }^{d}-x_{i}^{d}\right) \\
& x_{i}^{d}=x_{i}^{d}+v_{i}^{d}
\end{aligned}
$$

Where

$$
\begin{aligned}
& x_{i}: \text { the position of the ith particle } \\
& v_{i}: \text { the velocity of particle } i
\end{aligned}
$$

pbesti : the best location in the search space ever visited by particle $i$

\section{gbest : the best location discovered so far}

$\boldsymbol{w}:$ the inertia weight that controls the impact of previous velocity of particle on its current one

$\boldsymbol{r}_{1}, \boldsymbol{r}_{2}$ : independently uniformly distributed random variables with range $(0,1)$

$c_{1}, c_{2}$ : positive constants(acceleration) coefficients which control the maximum step size

Equation (7) is used to calculate the new velocity according to its previous velocity and to the distance of its current position from both its own best historical position and the best position of the entire population or its neighbourhoods. Generally, the value of each component in $v$ can be clamped to the range [-vmax, $v \max ]$ to control excessive roaming of particles outside the search space. Then the particle flies toward a new position. This process is repeated until a user-defined stopping criterion is reached. A linearly decreasing inertia weight from maximu $\mathrm{m}$ value wmax to min imu $\mathrm{m}$ value wmin is used to update the inertia weight:

$$
w^{k}=w_{\max }-\frac{w_{\max }-w_{\min }}{k_{\max }} \cdot k
$$

Kmax is maximum iteration number [16, 17].

\section{HBMO Algorithm}

The honey bee is a social insect that can only survive as a member of a community, or colony. The colony inhabits an enclosed cavity. The honey bee community structurally consists of three different forms: the queen (reproductive female), the drone (male), and the worker (non reproductive female). These castes are associated with different functions in the colony; each caste possesses its own special instincts geared to the needs of the colony. The behaviour of honey-bees shows many features like cooperation and communication, so honey-bees have aroused great interests in modeling intelligent behaviour these years. Marriage in honey-bees optimization (MBO) is a kind of swarm-intelligence method. Such swarm intelligence has some successful applications. Ant colony is an example and the search algorith $\mathrm{m}$ is inspired by its behaviour. Mating behaviour of honey-bees is also considered as a typical swarm-based optimization approach. The behaviour of honey-bees is related to the product of their genetic potentiality, ecological and physiological environ ments, the social conditions of the colony, and various prior and ongoing interactions among these three [18, 19]. The HBMO algorith $m$ combines number of different procedures. A drone mates with a queen probabilistically using an annealing function as follows [18, 20]:

$$
\operatorname{prob}(Q, D)=e^{\frac{-\Delta(f)}{s(t)}}
$$

where Prob(D) is the probability of adding the sperm of drone $\mathrm{D}$ to the spermatheca of the queen, $\Delta$ is the absolute difference between the fitness of $\mathrm{D}$ and the fitness of the queen and $S(t)$ is the speed of the queen at time $t$. The probability of mating is high when the queen is with the high speed level, or when the fitness of the drone is as good as the queen's. After each transition in space, the queen's speed decreases according to the following equations:

$$
s(t+1)=\alpha \cdot s(t) \quad 0<\alpha<1
$$

Where ${ }^{\propto}$ is the a mount of speed and energy reduction after each transition and each step. Initially, the speed of the queen is generated at random. A number of mating flights are realized. At the start of a mating flight drones 
are generated randomly and the queen selects a drone using the probabilistic rule in Eq. (10). If the mating is successful (i.e. the drone passes the probabilistic decision rule), the drone's sperm is stored in the queen's spermatheca. By using the crossover of the drone's and the queen's genotypes, a new brood (trial solution) is generated, which can be improved later by e mploying workers to conduct local search. One of the major differences of the HBMO algorithm from the classic evolutionary algorithms is that since the queen stores a number of different drone's sperm in her spermatheca, she can use parts of the genotype of different drones to create a new solution which gives the possibility to have fittest broods more. In real life, the role of the workers is restricted to brood care and for this reason the workers are not separate me mbers of the population and they are used as local search procedures in order to improve the broods produced by the mating flight of the queen. Each of the workers has different capabilities and the choice of two different workers may produce different solutions. This is realized with the use of a number of single local search heuristics $\left(N_{\text {workerl }}\right)$ and combinations of them $\left(N_{\text {worker2 }}\right)$ Thus, the sum of these two numbers $\left(N_{\text {worker }}=N_{\text {workerl }}+N_{\text {workee } 2}\right)_{\text {gives the number }}$ of workers. Each of the broods is randomly chosen to be feed by worker, which is also randomly selected. If the new brood is better than the current queen, it takes the place of the queen. If the brood fails to rep lace the queen, then in the next mating flight of the queen this brood will be one of the drones. Honey Bee Mating Optimization (HBMO) algorith $\mathrm{m}$ can be described briefly in 5 steps as below:

Step 1: This algorithm starts with flying in which the queen (the best answer) randomly selects its male pairs to consequently reproduce new bees.

Step 2: the new born bees (possible answers) will be reproduced by displacing in queen and male genes.

child $=$ parent $1+\beta \cdot($ parent $2-$ parent 1$) \quad 0 \leq \beta \leq 1$

Step 3: worker bees (research functions) are used to do the position seeking (breeding and improving new bee's generation).

$$
\begin{aligned}
& \operatorname{Brood}_{i}^{k}=\operatorname{Brood}_{i}^{k} \mp(\delta+\varepsilon) \cdot \operatorname{Brood}_{i}^{k} \\
& 0<\varepsilon<1 ; 0 \leq \delta \leq 1
\end{aligned}
$$

Step 4: Fitting functions of the workers will be sorted based on the amount of bees' generation improvement.

Step 5: the best new born bee in this process can be nominated to take queen position and do the next flying process.

\section{PSO\&HBMO Procedure}

In this article a new optimization method is presented based on the mixture of PSO and HBMO methods.
Players and motion speed is calculated through PSO relations in this method and choosing or suitability is obtained from HBMO method. Suitability analysis is performed using prob. function in HBMO method. In (10) $\mathrm{S}(\mathrm{t})$ is equal to particle speed in PSO method and $\Delta(f)$ can be calculated as below:

$$
\Delta(f)=\operatorname{gbest}(\text { it },:)-\text { particleposition }(i)
$$

Where gbest(it,:) is the best amount in all conditions and particle position (i) is the present position of the particle. We want to improve voltage profile and reduce power loss in this system by using 1,2 and 3 DG. Before installation of DG, load flow will be applied. Fig.2 shows voltage profile before DG installation. Fig.3 shows the results of sensitivity analysis.

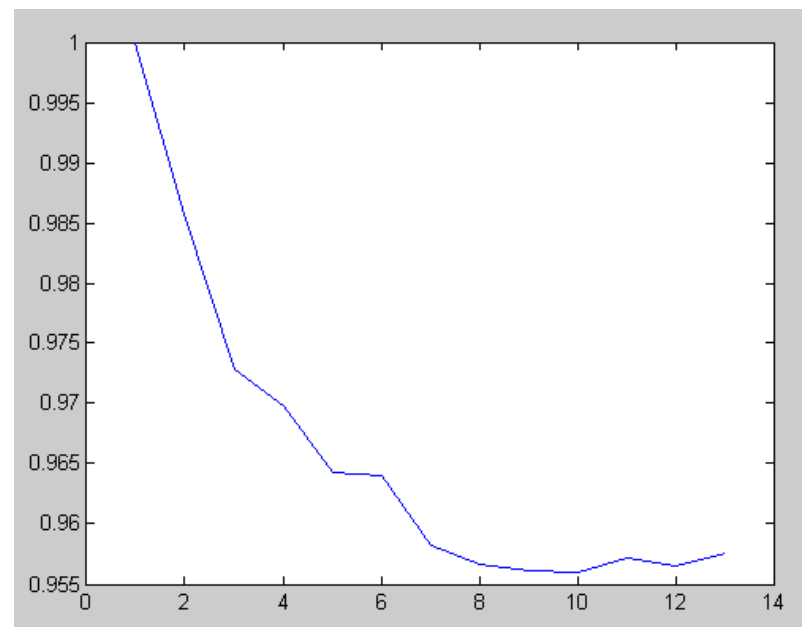

Fig. 2 Voltage profile before installation of DG

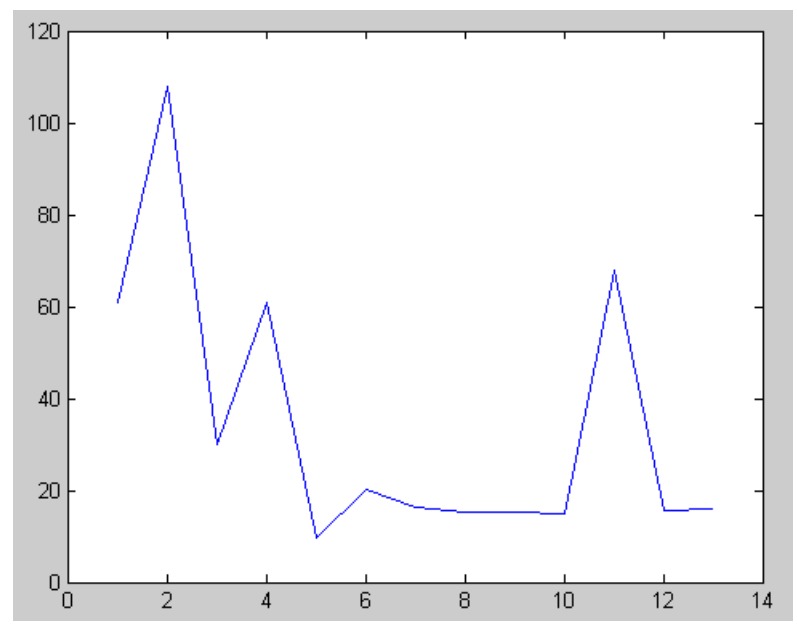

Fig. 3 Sensitivity of objective function to the location of DG

\section{Simulation Results}

The test system is radial distribution system with 13 buses as shown in Figure 4. The original total real power loss and reactive power and variance of voltage in the system are $336.24 \mathrm{~kW}, 264.01 \mathrm{kVar}$ and $0.45 \mathrm{pu}$, respectively. The min imu m and maximu $\mathrm{m}$ voltages are set 
at 0.95 and 1.05 p.u. respectively. The load data is given in Table 1 and branch data in is given Table2. Maximu $m$ number of DG is 3 . The maximu $\mathrm{m}$ real power and reactive power of DG are $1200 \mathrm{kw}$ and $5 \mathrm{kvar}$, respectively. The improvement in the voltage profile after optimally placing the DGs is shown in Figures 5 and 6. Without DG, the bus 10 has 0.955 p.u. and the bus voltage has improved to $0.9576 \mathrm{p}$.u. after installing DG. According to buses voltage profile, in PSO\&HBMO method voltage profile is better improved. Table 3 shows the simulation results of PSO and PSO\&HBMO methods for 13 bus systems. For the 13bus system, one DG can reduce the total power loss and variance of voltage and amount of objective function more than PSO algorithm. For two and three DGs, they can further reduce the total power loss and variance of voltage and amount of objective function more than PSO algorithm.

Table 1 Load data

\begin{tabular}{lll}
\hline BUS & $\mathbf{P}(\mathbf{K W})$ & $\mathbf{Q}(\mathbf{K V A R})$ \\
\hline 1 & 0 & 0 \\
2 & 890 & 468 \\
3 & 628 & 470 \\
4 & 1112 & 764 \\
5 & 636 & 378 \\
6 & 474 & 344 \\
7 & 1342 & 1078 \\
8 & 920 & 292 \\
9 & 766 & 498 \\
10 & 662 & 480 \\
11 & 690 & 186 \\
12 & 1292 & 554 \\
13 & 1124 & 480 \\
\hline
\end{tabular}

Table 2 Branch data

\begin{tabular}{llll}
\hline from & to & $\mathbf{R}$ & $\mathbf{X}$ \\
\hline 1 & 2 & 0.176 & 0.138 \\
2 & 3 & 0.176 & 0.138 \\
3 & 4 & 0.045 & 0.035 \\
4 & 5 & 0.089 & 0.069 \\
5 & 6 & 0.045 & 0.035 \\
5 & 7 & 0.116 & 0.091 \\
7 & 8 & 0.073 & 0.073 \\
8 & 9 & 0.074 & 0.058 \\
8 & 10 & 0.093 & 0.093 \\
7 & 11 & 0.063 & 0.05 \\
11 & 12 & 0.068 & 0.053 \\
7 & 13 & 0.062 & 0.053 \\
\hline
\end{tabular}

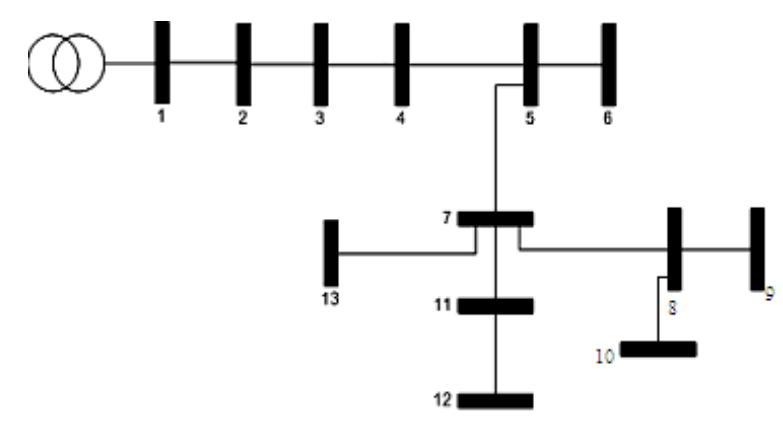

Fig. 4 The 13 bus radial distribution system

Table 3 Optimal DG placement and sizing

\begin{tabular}{|c|c|c|c|c|c|c|c|}
\hline $\begin{array}{l}\text { Objective } \\
\text { function }\end{array}$ & $\begin{array}{l}\text { Voltage } \\
\text { variance }\end{array}$ & $\begin{array}{l}\text { QL(KVA } \\
\text { R) }\end{array}$ & PL(KW) & DG size & Bus & $\begin{array}{l}\text { NUMBR } \\
\text { of DG }\end{array}$ & \\
\hline --- & 0.45 & 264.01 & 336.24 & 0 & 0 & 0 & Without DG \\
\hline 14.203 & 0.44 & 261.61 & 333.18 & $147+4.64 j$ & 2 & 1 & \multirow{3}{*}{ PSO } \\
\hline 15.62 & 0.43 & 248.041 & 315.90 & $\begin{array}{l}981.72+3.07 \mathrm{j} \\
909.71+2.84 \mathrm{j}\end{array}$ & 2,11 & 2 & \\
\hline 11.88 & 0.43 & 243.32 & 309.86 & $\begin{array}{l}1335.4+4.2 \mathrm{j} \\
278.1+0.9 \mathrm{j} \\
550.9+1.7 \mathrm{j}\end{array}$ & $2,11,4$ & 3 & \\
\hline 11.57 & 0.42 & 240.68 & 306.48 & $1572.3+4.91 \mathrm{j}$ & 2 & 1 & \multirow{3}{*}{ PSO\&HBMO } \\
\hline 10.75 & 0.42 & 242.041 & 308.23 & $\begin{array}{l}1386.60+4.3 \mathrm{j} \\
1436.1+4.5 \mathrm{j}\end{array}$ & 2,11 & 2 & \\
\hline 9.84 & 0.42 & 240.42 & 306.15 & $\begin{array}{l}1564.9+4.9 \mathrm{j} \\
306.8+1 \mathrm{j} \\
1313.3+0.4 \mathrm{j}\end{array}$ & $2,11,4$ & 3 & \\
\hline
\end{tabular}




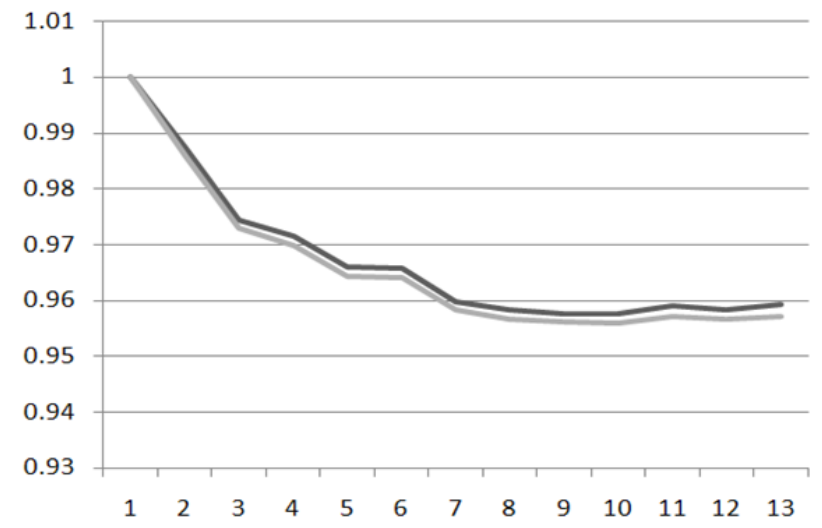

Fig.5 Three DG installing (upper), Without DG (lower)

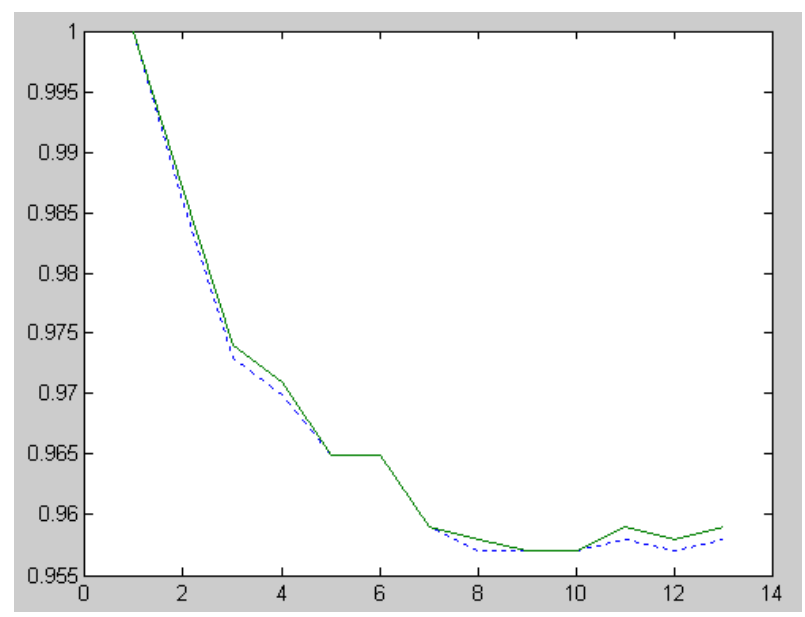

Fig 6 Voltage profile of 2 DGs, PSO (dash line) and PSO\&HBMO (solid line)

\section{Conclusions}

The Distributed Generation (DG) in a distribution system offers several benefits such as relieved transmission and distribution congestion, voltage profile improvement, line loss reduction, improvement in the system, and enhanced utility system reliability. This proposed work has presented an approach to quantity some of the benefits of DG namely, real power loss reduction and voltage profile improvement of system. In this paper, a hybrid algorith $m$ PSO\&HBMO for optimal placement and sizing of multi-DGs proposed. This method efficiently minimize the total power loss and variance of voltage and a mount of objective function and improve the voltage profile satisfying transmission line limits and constraints. The methodology is accurate in determining the sizes and DG locations.

\section{References}

[1] T. Ackermann, G. Anderson, L. Söder; "Distributed Generation: a Definition", Electric Power System Research, 2001, Vol. 57, pp.195-204.
[2] P. P. Barker, R. W. de Mello; "Determining the Impact of Distributed Generation on Power Systems: Part 1-Radial Distribution Systems", IEEE PES Su mmer Meeting, 2000, Vol.3, pp.1645-1656.

[3] N. Hadjsaid, J. F. Canard, F. Dumas; "Dispersed Generation Impact on Distribution Networks", IEEE Compt. Appl. Power, 1999, Vol. 12, pp.22-28.

[4] W.El-Khattam, M.M.A.Salama; "Distributed Generation Technologies, Definitions and Benefits", Electric Power Systems Research, 2004, Vol. 71, pp.119-128.

[5] G.Pepermans, J.Driesen, D.Haeseldonckx, R.Belmans, W.D.haes eleer; "Distributed Generation: Definitions, Benefits and Issues", Energy Policy, 2005, Vol. 33, pp.787-798.

[6] A.Soroudi, M.Afrasiab; "Binary PSO-Based Dynamic Multi-Objective Model for Distributed Generation Planning under Uncertainty", IET Renewable Power Generation, 2012, Vol.6, No. 2, pp. $67-78$.

[7] Kai Zou, A.P.Agalgaonkar, K.M.Muttaqi, S.Perera; "Distribution System Planning with Incorporating DG Reactive Capability and System Uncertainties" ,IEEE Transactions on Sustainable Energy, 2012, Vol.3 , No.1, pp. 112 - 123.

[8] Sheng-Yi Su, Chan-Nan Lu, Rung-Fang Chang, G. Gutiérrez-Alcaraz; "Distributed Generation Interconnection Planning: A W ind Power Case Study ", IEEE Transactions on Smart Grid, 2011, Vol.2 , No.1, pp. 181 - 189.

[9] Ruifeng Shi, Can Cui, Kai Su, Zaharn Zain; "Comparison Study of Two Meta-heuristic Algorith ms with their Applications to Distributed Generation Planning", Energy Procedia, 2011, Vol. 12, pp. 245-252.

[10] A.M. El-Zonkoly; "Optimal Placement of Multi-Distributed Generation Units Including Different Load Models using Particle Swarm Optimization", Swarm and Evolutionary Computation, 2011, Vol.1, No.1, pp. 50-59.

[11] M.R. AlRashidi, M.F. AlHajri; "Optimal Planning of Multiple Distributed Generation Sources in Distribution Networks: A New Approach", Energy Conversion and Management, 2011, Vol. 52, No. 11, pp. 3301-3308.

[12] M.H. Moradi, M. Abedini; "A Combination of Genetic Algorithm and Particle Swarm Optimization for Optimal DG Location and Sizing in Distribution Systems", International Journal of Electrical Power \& Energy Systems, 2012, Vol.34, No.1, pp. 66-74.

[13] Qi Kang, Tian Lan, Yong Yan, Lei Wang, Qidi Wu; "Group Search Optimizer Based Optimal Location 
and Capacity of Distributed Generations", Neurocomputing, 2012, Vol.78, No.1, pp. 55-63.

[14] M. Gomez-Gonzalez, A. López, F. Jurado; "Optimization of Distributed Generation Systems using a New Discrete PSO and OPF", Electric Power Systems Research, 2012, Vol.84, No.1, pp. 174-180.

[15] R. Srinivasa Rao; "Capacitor Placement in Radial Distribution System for Loss Reduction using Artificial Bee Colony A lgorith m", World Acade my of Science, Engineering and Technology, 2010, Vol. 68.

[16] Y. Wang, B. Li, T. Weise, J. Wang, B. Yuan, Q. Tian; "Self-Adaptive Learning Based Particle Swarm Optimization", Information Sciences, 2011, Vol.181, No.20, pp. 4515-4538.

[17] Y. Jiang, T. Hu , C. Huang, X. Wu; “An Improved Particle Swarm Optimization Algorithm”, Applied Mathematics and Computation, 2007, Vol. 193, No.1, pp. 231-239.

[18] M. Fathian, B.A miri, A. Maroosi; "Application of Honey-Bee Mating Optimization Algorithm on Clustering", Appl. Math. Comput., 2007, 190(2), pp.1502-1513.

[19] A. Afshar, O. Bozorg Haddad, M.A. Mariº, BJ.Adams; "Honey-Bee Mating Optimization (HBMO) Algorithm for Optimal Reservoir Operation", J. Franklin Inst., 2007, 344(5), pp.452-462.

[20] T. Niknam; "Application of Honey Bee Mating Optimization on Distribution State Estimation Including Distributed Generators", J. Zhejiang Univ. Sci. A, 2008, 9(12), pp.1753-1764.

\section{Authors' Profiles}

Mohammad Afzalan was born in Ahvaz, Iran, in 1987. He received the B.Sc. and M.Sc. degrees in electrical engineering from Azad University, Iran. His interests are power system, distribution networks and power quality.

Mohammad Ali Taghikhani was born in Tehran, Iran, in 1974. He received the B.Sc. and M.Sc. degrees in electrical engineering from Amirkabir University of Technology, Tehran, Iran, in 1997 and 2000, respectively. He received the Ph.D. degree in electrical engineering from Iran University of Science and Technology, Tehran, Iran, in 2008. He was with the faculty of Azad University, Tehran, Iran. He is an assistant professor and currently with the faculty of Imam Khomeini International University, Qazvin, Iran. His interests are power system, numerical analysis, fin ite ele ment method, heat transfer and fluid mechanics.
How to cite this paper: M.Afzalan, M. A.Taghikhani,"Placement and Sizing of DG Using PSO\&HBMO Algorithms in Radial Distribution Networks", International Journal of Intelligent Systems and Applications(IJISA), vol.4, no.10, pp.43-49, 2012. DOI: 10.5815/ijisa.2012.10.05 